\title{
Comprehensive transcriptomic analysis of Papillary Thyroid Cancer: potential biomarkers associated with tumor progression
}

\section{Nazanin Hosseinkhan}

Institute of Endocrinology and Metabolism, Iran University of Medical Sciences

Maryam Honardoost

Institute of Endocrinology and Metabolism, Iran University of Medical Sciences

Kevin Blighe

Biomedical Sciences Research Institute, Ulster University

C.B. Tara Moore

Biomedical Sciences Research Institute, Ulster University

Mohammad Ebrahim Khamseh ( $\nabla$ khamseh.m@iums.ac.ir)

Institute of Endocrinology and Metabolism,Iran University of Medical Sciences https://orcid.org/00000003-4313-8440

\section{Research article}

Keywords: Papillary Thyroid Cancer, tumor progression, patient stratification, biomarkers

Posted Date: December 10th, 2019

DOl: https://doi.org/10.21203/rs.2.15631/v2

License: (c) (1) This work is licensed under a Creative Commons Attribution 4.0 International License.

Read Full License

Version of Record: A version of this preprint was published at Journal of Endocrinological Investigation on January 21st, 2020. See the published version at https://doi.org/10.1007/s40618-019-01175-7. 
The authors have withdrawn this preprint from Research Square 